Les dictionnaires bilingues des maîtres ou professeurs auteurs de manuels pédagogiques

\title{
La découverte du français non-conventionnel outre-Rhin: le dictionnaire des Parisismen de Césaire Villatte (1816-1895)
}

Franz-Joseph Meissner

\section{(Q) OpenEdition}

\section{Journals}

Édition électronique

URL : https://journals.openedition.org/dhfles/3957

DOI : $10.4000 /$ dhfles.3957

ISSN : 2221-4038

Éditeur

Société Internationale pour l'Histoire du Français Langue Étrangère ou Seconde

Édition imprimée

Date de publication : 1 juin 2016

Pagination : 103-124

ISSN : 0992-7654

Référence électronique

Franz-Joseph Meissner, «La découverte du français non-conventionnel outre-Rhin: le dictionnaire des Parisismen de Césaire Villatte (1816-1895) », Documents pour l'histoire du français langue étrangère ou seconde [En ligne], 56 | 2016, mis en ligne le 04 février 2018, consulté le 27 mars 2023. URL : http:// journals.openedition.org/dhfles/3957 ; DOI : https://doi.org/10.4000/dhfles.3957

Ce document a été généré automatiquement le 27 mars 2023.

Tous droits réservés 


\title{
La découverte du français non- conventionnel outre-Rhin: le dictionnaire des Parisismen de Césaire Villatte (1816-1895)
}

\author{
Franz-Joseph Meissner
}

\begin{abstract}
«L'argot ne doit pas être parlé, mais il doit être connu. »
Clément Casciani (1894:47)
\end{abstract}

$1 \mathrm{Au} \mathrm{XIX}^{\mathrm{e}}$ siècle, l'enseignement-apprentissage des langues étrangères outre-Rhin, s'intègre dans la démocratisation de l'éducation publique. L'évolution est l'expression de l'avènement de la société moderne : industrialisation, apparition des grandes villes en Allemagne : Duisbourg, Essen, Dortmund, Munich, Berlin..., de professions nouvelles (employés, ouvriers industriels) et disparition d'autres, renouveau des universités et essor des sciences, organisation de l'administration étatique moderne, standardisation de la formation professionnelle dont celle des enseignants de langues, contrôle administratif des établissements scolaires et de leurs activités pédagogiques, etc.

Dans une Allemagne de plus en plus industrialisée, tous les enfants sont soumis à l'éducation scolaire (Allgemeine Schulpflicht) et l'analphabétisme finit par diminuer considérablement. Le français s'enseigne dans les Gymnasien (lycées), Lyzeen (lycées pour jeunes filles), Realgymnasien et Realschulen (établissements orientés davantage vers la vie pratique). Les cours de langues atteignent entre $2 \%$ ou $3 \%$ et $10 \%$ de la population scolaire.

\section{Enseignants, enseignement et le français parlé au $\mathrm{XIX}^{\mathrm{e}}$ siècle}

3 À partir de 1830, date de la nomination de Friedrich Diez à la première chaire de philologie romane (Bonn), cette discipline naissante commence à se charger de la formation des enseignants de français qui remplacent petit à petit les anciens 
«maîtres » de langue (Christ 2005). L'éducation de ces professeurs est basée sur l'étude des littératures romanes et d'une linguistique (elle aussi en voie de naître) orientée sur la diachronie. Même si ces études sont doublées de l'apprentissage pratique d'une ou deux langues romanes, les compétences acquises servent plutôt à donner des ressources nécessaires pour atteindre les objectifs "scientifiques" (littéraire et linguistique). La communication pratique en langue-cible ne figure pas encore parmi les objectifs pédagogiques primordiaux ${ }^{1}$. En même temps, les lecteurs francophones (natifs) sont de plus en plus rares. Quant aux conséquences de cette évolution, Schröder (1989: 62) observe que dans l'Allemagne d'entre 1820 et 1880, on rencontre trois générations de lycéens, enseignants, professeurs des universités qui parlent un français que Wilhelm Viëtor, figure de proue du Mouvement de la Réforme (Reformbewegung), décrira dans ces vers moqueurs :

Sprecht ihr aber doch Französisch,

solls nicht lauten wie Chinesisch,

Träng, Detalch und Reglemang

ist ein sonderlicher Klang. (Viëtor $1882: 61)^{2}$

Dans les écoles allemandes de l'époque postnapoléonienne, un certain déclin du français va de pair avec l'essor du nationalisme allemand. Même comme matière classique de l'éducation formelle (formale Bildung), le français recule jusqu'à être banni des lycées (Gymnasien) prussiens entre 1816 et 1830. En même temps, les francophones de naissance sont, là aussi, souvent remplacés par des enseignants d'origine allemande, surtout des hommes «qui ajoutent à une connaissance profonde de la langue et littérature françaises la maîtrise aisée de l'allemand (la langue maternelle), une éducation classique et une bonne mentalité scolaire » (d'après Schröder 1989 : 37 ; trad. par F-JM.).

5 Tandis que l'enseignement des anciens maîtres avait visé surtout la préparation des élèves à la communication orale et écrite avec les locuteurs natifs en langue-cible, l'enseignement scolaire poursuivait d'autres objectifs. Les Instructions pour Jeunes Filles de Hambourg stipulent encore en 1910, c'est-à-dire après la réorientation de l'enseignement, l'objectif pédagogique général comme suit :

Compréhension d'ouvrages pas trop difficiles, surtout de la littérature actuelle, maîtrise de la traduction stylistiquement adéquate, capacité à la lecture en autonomie, connaissance de la vie et des mœurs des deux peuples (français et anglais, F-JM.), prononciation correcte, familiarisation avec des phénomènes grammaticaux, habilitation à se servir de la langue étrangère au niveau de l'écrit et de l'oral. (Christ \& Rang 1985 : II, 99 ; trad. par F-JM.)

6 Les Instructions pour les Höhere Bürgerschulen (Gymnasien) du 31-3-1882 vont dans le même sens :

Bonne prononciation, capacité à la lecture, connaissance pratique de la morphologie et des règles de la syntaxe, appropriation d'un lexique suffisamment riche pour la lecture scolaire. Exercices pour reproduire un texte en français ou en anglais, lecture d'une prose facile, surtout de caractère descriptif ou historique et de poèmes simples. (Christ \& Rang 1985 : II, 67 ; trad. par F-JM.)

7 Bonne prononciation, connaissance de la morphologie, de la syntaxe et d'un vocabulaire orienté sur la lecture scolaire ainsi qu'une certaine familiarité avec les us et coutumes de la culture-cible, à savoir institutions et histoire françaises - tels étaient, somme toute, les objectifs pédagogiques de l'époque. - La compétence orale en languecible comme objectif pédagogique n'en était pas totalement absente. Mais évidemment, l'usage du terme reflète une acception naïve qui ne faisait pas le tri entre un canal 
(graphique, acoustique) et la planification (écrit [élaboré], parlé [spontané]). Cet usage amène à jeter un regard sur la philologie romane et sa branche linguistique de l'époque, formatrice des enseignants de français et d'autres langues néolatines.

Cette philologie se focalisait sur des phénomènes historiques. Pour ce qui est de la linguistique, on mentionnera: évolution des langues romanes, étymologie, sémasiologie et onomasiologie, phonétique historique et la confection de grandes encyclopédies étymologiques très réputées. Somme toute, la langue parlée ne figurait pas encore parmi ces intérêts scientifiques.

9 La situation change quand la linguistique découvre la langue contemporaine. À l'égard des pays germanophones, Greive (1984) a retracé l'évolution de cet intérêt de Leo Spitzer et de ses ouvrages Italienische Kriegsgefangenenbriefe (1921), Italienische Umgangssprache (1922) à Werner Beinhauer Frases y diálogos de la vida diaria (1925), Spanische Umgangssprache (1930) et à Ludwig Söll Gesprochenes und geschriebenes Französisch (Söll \& Hausmann 1974, 1985). Pour le français, on mentionnera la thèse de doctorat d'August Prein Syntaktisches aus französischen Soldatenbriefen (Giessen, 1921). Prein, qui pendant la Première Guerre mondiale était chargé de "contrôler" la correspondance de prisonniers de guerre, constate un écart important entre le français décrit dans les grammaires de français allemandes et la réalité de cette langue, dans les lettres des soldats. En découvrant un hiatus considérable entre les langues grammaticographiées sur la base de l'écrit et les parlers tels qu'ils se manifestaient dans le langage quotidien des natifs, Prein, Spitzer, Beinhauer et d'autres se trouvent (sans doute à leur insu) un peu dans le sillage de Césaire Villatte.

\section{Césaire Villatte}

10 Né le 13 janvier 1816 à Neu-Strelitz où son père, après avoir quitté la France lors de la Révolution, enseignait le français et où le petit Césaire passe son enfance, Villatte fait des études classiques à Berlin. Après le doctorat, il séjourne en France. En 1838, Villatte entre au Collegium Carolinum de Neu-Strelitz, où il enseignera le français pendant 45 ans « avec grand succès » (selon son biographe Ludwig Fränkel en 1895). Peu avant sa retraite en 1883, il obtiendra le titre de professeur. Il meurt en 1895.

11 En tant que co-directeur (au côté de Karl Sachs) du grand Enzyklopädisches französischdeutsches und deutsch-französisches Wörterbuch (Berlin-Schöneberg : Langenscheidt 1865), Villatte connaît une certaine célébrité. Cet ouvrage, publié par le libraire berlinois Gustav Langenscheidt, sera considéré, dans les pays germanophones pour de nombreuses décennies, comme le chef d'œuvre de la lexicographie bilingue françaisallemand / allemand-français. Dès la première édition, Villatte rédige un grand nombre d'articles concernant notamment le français parlé ou le français quotidien. L'année 1884 voit la parution des «Parisismen. Gründliches alphabetisches Verzeichniß des Pariser Argot ». Ce dictionnaire bilingue connaît un vaste écho et comptera, jusqu'en 1912, huit éditions (refontes et mises à jour). 


\section{Aux origines de la perception du français non- conventionnel (argot, familier, vulgaire, etc.) en Allemagne}

Qui veut décrire le registre parlé d'une langue nationale se voit confronté à une infinité de variétés diatopiques, diastratiques et diaphasiques parmi lesquelles en choisir une comme objet de description (Meissner 2008). Quant au français et à cet embarras du choix, les auteurs donnent - unanimement - la préférence au parler populaire de Paris. Bauche (1920) en explique les principales raisons comme suit :

Le français populaire de Paris est (...) le français populaire de toute la France, de la France, du moins, qui parle français. (Ibid. : 183)

Argot, familier, populaire, vulgaire, jargon, loucherbem, verlan, branché (toujours aux couleurs de Paris), ce sont là tous des termes désignant différents registres d'un français non-conventionnel ou substandard (Cellard \& Rey 1981). Il s'agit de registres peu définis et peu distincts. Leur dénominateur commun découle de leur appartenance primordiale à la langue parlée, ce qui n'empêche pas que, depuis un Victor Hugo ou un Emile Zola, une oralité fictionnelle incorpore des mots non-conventionnels à la langue écrite. Notons que des auteurs de notre époque comme le journaliste Alain Schifres y ajoutent d'autres désignations parfois assez originales comme l'adoparisien («mots des jeunes Parisiens qu'aiment employer les vieux Parisiens pour oublier un instant qu'ils vont crever ») et le parigomondain ("mots grossiers [du genre qui sert à écrire en langage parlé] et de mots affectés [... qui sert à parler en langage écrit »]) (1990: 201). Que la lexicographie traditionnelle ait surtout été une "lexicographie du bon usage », basée sur les œuvres des bons auteurs (donc sur l'écrit), allait souvent de pair avec un certain mépris pour la langue populaire, parlée par le peuple (le bas langage, les prétendus argots, etc.). D'où, pendant longtemps, une rareté relative de glossaires enregistrant ces parlers dont Esnault (1965: VIII) a dressé un bref historique lexicographique partant de la bibliographie de l'argot d'Yves Plessis (1901). À l'égard des Parisismen, on retiendra que la deuxième moitié du XIXe siècle connaît pourtant un évident essor de la production de dictionnaires dédiés à ce registre souvent méprisé et admiré en même temps (Larchay 1858, Delvau 1866, Rigaud 1878, La Rue 1894, Virmaitre 1894, Delesalle 1895, Bruant 1901 - presque tous mentionnés par Esnault). Mais, bien sûr, il s'agit là toujours de dictionnaires qui s'adressent à un public francophone et adulte.

en Arançais non-conventionnel en Allemagne et de son enseignementapprentissage n'a pas encore été écrite. Au moins six raisons peuvent être invoquées :

Comme le vocabulaire non-conventionnel appartient profondément à la langue parlée et comme ses dictionnaires enregistrent une réalité linguistique sonore (et fugace), il a échappé à une lexicographie construite à partir de sources écrites. Par conséquent, ce registre n'était que difficilement saisissable pour l'enseignement du français à l'étranger.

Une fois établi comme l'instrument pédagogique primordial au service d'un «bon usage » qui se confondait souvent avec le bon goût tout court, le dictionnaire n'accorde pas forcément droit de cité aux mots populaires considérés comme une quantité négligeable. Néanmoins, leur exclusion ne satisfaisait qu'une partie des destinataires désireux de trouver une description plus parfaite de leur langue de tous les jours. Quant à ce clivage, Cellard \& Rey (1981 : IX) observent : 
[...] ils (les mots «négligés ») manifestent par leur nombre et leur vitalité que la langue n'est pas 'une' et unie sous la férule paternelle des Académiciens et des Ecoles. C'est cela au fond, qui n'a jamais été pardonné au français nonconventionnel [...]

\section{linguistique qui est celle d'une bourgeoisie disposant des compétences et moyens d'une} culture dont le prestige est fondé sur une tradition scripturale : les ouvrages de grands auteurs, les belles lettres, la pensée philosophique, etc. Elle est en revanche beaucoup moins celle du peuple, privé des privilèges découlant de ces compétences et moyens. À regarder le profil sociologique des apprenants germanophones du français (ou de l'anglais), on découvre, là aussi, un public plus bourgeois que populaire. À la rigueur, on pourrait être tenté de parler d'une sorte de "complicité » entre les couches sociales privilégiées française et allemande ${ }^{3}$.

Aux yeux du romaniste influent Karl Vossler (1872-1945), qui a pour disciples Viktor Klemperer, Eugen Lerch et Werner Krauss, le français parlé quotidiennement ne paraît pas "digne » d'être enseigné aux élèves. Ainsi dit-il à l'occasion de la $18^{\mathrm{e}}$ Journée des Philologues Modernes (Neuphilologen-Tag), le 7 juin 1922 à Nuremberg : acceptable entre la norme prétendue du bon usage et la norme statistique d'un français palpable dans l'oralité de la vie quotidienne hexagonale, il n'en est pas ainsi dans les territoires coupés de l'oralité naturelle du français. Dans une époque sans internet, sans télévision par satellite, sans possibilité de capter à n'importe quel endroit n'importe quelle radio en n'importe quelle langue, les manifestations authentiques du français parlé sont réduites au fur et à mesure qu'elles sont véhiculées par les journaux et les belles lettres ou - bien sûr - par les dictionnaires. Autrement dit, le français parlé « captable » à l'étranger est du type « parlé scriptural » faisant abstraction d'éléments visuels et de l'implication de l'apprenant dans toute situation communicative authentique. Aussi n'apprend-il jamais la complémentarité entre les codes verbal et extra-verbal. Dans sa forme acoustique authentique, le français parlé - qu'il soit populaire ou non - reste aux yeux et à l'oreille de l'apprenant une terra incognita ${ }^{4}$. Fautil rappeler qu'on apprend les parlers à force de les écouter et leur intégration dans une situation communicative donnée à force de voir et d'écouter?

Alors que la fugacité de la parole sonore et l'absence presque totale de la complémentarité entre l'image acoustique et visuelle de la langue représentaient 
longtemps un des obstacles les plus importants pour l'apprentissage d'une langue parlée moderne, cet obstacle est devenu surmontable grâce aux TICES et à leurs multiples fonctions. Il va sans dire que ce changement exige un enseignement approprié.

Ce qui manquait à l'époque de Villatte, c'était une description plus complète du français, comme on la trouve dans l'ouvrage d'Henri Bauche Le langage populaire. Grammaire, syntaxe et dictionnaire du français tel qu'on le parle dans le peuple de Paris avec tous les termes d'argot usuel (1920). Celui-ci traite de la prononciation, la formation des mots, les genres, les nombres, les articles, les substantifs, les adjectifs, les pronoms, le verbe, l'adverbe, la préposition, la conjonction, l'interjection, les jurons (sic), les mots grossiers, les explétifs, la phrase populaire, les locutions diverses, l'orthographe, les formules de politesse, la parenté, la transformation de la langue. Le langage populaire est beaucoup plus explicite qu'un dictionnaire. Conformément à la question fondamentale de la sociolinguistique "qui parle - à qui - avec quelle intention - où - comment ", Bauche ne se contente pas d'enregistrer un lemme et d'en donner un petit co-texte illustrant l'usage si nécessaire. Regardons ce qu'il dit à propos de l'appellatif «Madame » tel qu'il s'utilise vers 1920:

...lorsqu'on parle à un homme de sa femme à lui, il serait inconvenant de dire « votre femme ». « Madame » suivi du nom du mari, est assez rare. « Madame » tout seul est rare également. On dit votre dame. Lorsqu'on tutoie la personne à qui on parle, on a le droit de dire « ta femme ». (Ibid. : 176)

Un autre exemple : la préposition qu'on emploie dans les relations de parenté [...] n'est pas « de », mais « à » : « la femme à Ugène, [...], le livre à Paul... » (ibid. : 148).

$\mathrm{Vu}$ que la lexicographie traditionnelle du non-conventionnel s'adressait exclusivement aux francophones, on s'étonne de l'attention que Bauche prête à l'apprenant étranger :

L'accent parisien n'est pas difficile à acquérir lorsqu'on connaît bien les règles générales de prononciation [...].

[...] la lettre la plus difficile à prononcer est l'r. Un Anglais, un Bourguignon, un Italien y réussissent difficilement. L'Espagnol, le Russe, l'Allemand, qui possèdent chacun dans sa langue la correspondante sourde de l'r, y parviennent plus vite. (Ibid. : 61)

\section{Le dictionnaire des « Parisismen »}

\subsection{Genèse et éditions}

La confection du dictionnaire des Parisismen se confond avec celle de l'Enzyklopädisches deutsch-französisches Wörterbuch, rédigé sous l'égide de Karl Sachs et auquel Villatte a contribué avec de nombreux articles concernant surtout le français parlé, ce qui, en fin de compte, a justifié son statut de co-directeur de ce projet important. Quant à la publication des Parisismen sous forme d'un dictionnaire indépendant, Villatte se voit dans l'obligation de s'expliquer. Dans l'avant-propos de la première édition du dictionnaire de 1884, il écrit :

Quand nous nous proposons de présenter un recueil des argotismes de Paris, c'est pour répondre à un véritable besoin, car, jusqu'à l'heure actuelle, de tous les dictionnaires français-allemand, seul l'Enzyklopädisches Wörterbuch de Sachs-Villatte, dans sa version complète, tient compte du véritable argot. Pour tous ceux qui ont l'intention de passer un certain temps à Paris, ce livre sera donc - je l'espère - 
bienvenu, afin de compléter les autres dictionnaires. (Villatte [1884], 1912: VII, trad. par F.-J. M.) "suppléments» dont le titre complet est: Parisismen, alphabetisch geordnete Sammlung der eigenartigen Ausdrucksweisen des Pariser Argot; ein Supplement zu allen französisch-deutschen Wörterbüchern. Sous cette forme, les Parisismen voient de nombreuses refontes et éditions: en 1884, 1888, 1890, 1895, 1899, 1906, 1912. Par rapport à leur différence à l'égard du dictionnaire indépendant du même nom, Villatte souligne :

Et surtout, il n'était pas possible de prendre suffisamment en considération les caractéristiques de l'argot parisien au niveau d'exemples illustratifs et d'explications, comme les intentions et l'espace des Parisismen, par contre, le permettent. (Ibid. : VII ${ }^{5}$, trad. par F.-J. M.)

Jusqu'en 1912, neuf éditions, refontes et mises à jour témoignent de l'intérêt remarquable que le public germanophone apporte au dictionnaire. La seule année 1912 voit deux éditions, révisées et augmentées par Rudolf Meyer-Riefstahl et Marcel Flandrin. En 1892, le dictionnaire connaît une version néerlandaise: Alphabetisch gerangschikte Verzameling der eigenaardige Zegswijzen van het parijsche Argot. Suppl. op alle fransch-nederlandsche Woordenboeken (Gouda : van Goor Zonen). destiné à un public étranger, souvent peu familiarisé avec la langue de Voltaire (formé par la littérature scolaire et peu enclin à intégrer la littérature « argotique » dans son canon de «bons auteurs») ? Comme ses homologues français, Villatte se réfère au décalage entre le français très fidèle à une norme prétendue et celui de la norme « statistique ", reflétée dans la langue parlée.

De nos jours, l'argot de Paris ne se confine pas seulement à la communication orale de toutes les couches sociales. Il s'est répandu petit à petit dans les journaux, les pièces de théâtre et, surtout et en grande quantité, dans les romans de l'école naturaliste. [...]. D'où la grande importance de l'argot. Ainsi, Victorien Sardou dit dans la Famille Benoiton : «L'argot est le français de l'avenir ». (Ibid. : IV, trad. par F.J. M.)

31 Nul doute alors que celui qui veut comprendre les vaudevilles, les romans des naturalistes, sans parler de la presse écrite en plein essor, a tout intérêt à se familiariser au moins à des fins réceptives avec la langue hexagonale telle qu'on la parle, à savoir à connaître les « parisianismes » :

En préparant la première édition des Parisismen, j'avais l'intention de permettre aux lecteurs allemands la compréhension de journaux, pièces de théâtre, romans, etc. Que l'objectif ait été atteint, a été prouvé par la grande estime que l'ouvrage a trouvée dans une soixantaine de comptes rendus. (Ibid.: Avant-propos de la $2^{\mathrm{e}}$ édition, trad. par F.-J. M.)

Évidemment, il a fallu attendre la linguistique de la deuxième moitié du $\mathrm{XX}^{\mathrm{e}}$ siècle pour libérer définitivement l'argot d'un faux romantisme dans lequel baignait le terme depuis plusieurs siècles et faire découvrir ce registre tel quel au grand public. En tant que lexicographe et enseignant du XIX ${ }^{\mathrm{e}}$ siècle, Villatte se voit encore dans l'obligation de prévenir les soupçons ou même les reproches d'avoir publié un glossaire de «mots sales ", enregistrant le langage des gens de mauvaise vie. D'où son exhortation: Attention, les Parisismen sont réservés aux adultes. 
Avant de clore, il semble à propos de prévenir: Ce livre n'a pas du tout été écrit pour la jeunesse apprenant le français, mais pour un public adulte. (Ibid., trad. par F.-J. M.)

Quelles sont les sources des Parisismen? Villatte ne les cache pas :

Comme la première édition ne contient à peine autre chose qu'un extrait des dictionnaires d'argot dans la mesure où je les ${ }^{6}$ connaissais, mon travail se bornait à la compilation et traduction en allemand du vocabulaire. (Ibid. : IX, trad. par F.-J. M.)

Pour la deuxième édition, révisée et augmentée, Villatte se sert de sources supplémentaires. Pour saisir le français parlé sur le vif, il parvient à gagner la collaboration de l'écrivain parisien Oscar Métenier, dont La Chair contient plusieurs textes entièrement composés dans l'argot des malfaiteurs. Métenier exerce une haute fonction dans la police parisienne, ce qui, aux yeux de Villatte, est une garantie pour l'authenticité du langage attribué. De plus, Villatte s'adjoint les services d'autres conseillers.

Après la mort de Villatte en 1895, Rudolf Meyer-Riefstahl se charge des $8^{\mathrm{e}}$ et $9^{\mathrm{e}}$ éditions des Parisismen, révisées et augmentées elles aussi. Né en 1880 à Munich, il rédige, en 1903, une thèse de doctorat à l'université de Strasbourg ${ }^{7}$, il enseigne l'allemand en Sorbonne. En 1911, il quitte la France pour se vouer surtout à l'étude de l'art islamique. D'autres étapes importantes de sa vie sont Londres, New York, Istanbul, Le Caire. C'est lors de son séjour à Paris que Meyer-Riefstahl fait la connaissance de Marcel Flandin, professeur agrégé au lycée de Clermont-Ferrand, lui aussi co-auteur de la refonte de la $8^{\mathrm{e}}$ édition.

L'avant-propos de ces éditions souligne la nécessité d'une remise à jour due à l'évolution rapide du registre pour laquelle Meyer-Riefstahl et Flandin mentionnent deux raisons principales : la créativité remarquable des parlers populaire et familier, à savoir l'augmentation de l'inventaire potentiel, et la rapidité avec laquelle les argotismes tombent en désuétude. Quant aux destinataires des Parisismen, les deux directeurs se voient amenés à crier " gare " :

Souvent, nous pouvons observer que les Allemands, à peine arrivés à Paris, sont fiers de chaque mot d'argot que leur mémoire a retenu, et qu'ils n'hésitent pas à utiliser. [...] Nous aimerions que ce dictionnaire soit compris comme une liste de mots que l'Allemand à Paris n'utilise pas. Car ce n'est que grâce à une familiarité avec le français acquise au fil de beaucoup d'années qu'on finit par connaître les nuances de l'argot. (Ibid. : XII, trad. par F.-J. M.)

\subsection{Macro- et microstructure des Parisismen}

En gros, la huitième édition du dictionnaire enregistre 12600 articles et trois, quatre ou même cinq fois plus de significations. Les enregistrements sont trop nombreux pour un dictionnaire d'apprentissage. Mais la maîtrise du vocabulaire, surtout à des fins de compétence productive, n'est dans l'intention ni de Villatte, ni de Meyer-Riefenstahl ou de Flandin. Les Parisismen visent exclusivement à la promotion d'une compétence de compréhension écrite ou orale.

Ni Villatte, ni Meyer-Riefenstahl ou Flandin ne s'expliquent en détail sur les principes qui dirigent la sélection des vocables enregistrés, à l'exception d'une constatation assez globale, concernant l'actualité du vocabulaire et du rejet de mots appartenant à ce qu'ils appellent « l'argot de fantaisie». 
La microstructure des articles suit plus ou moins le schéma suivant ${ }^{8}$ :

LEMME - catégorisation diaphasique ou diastratique - genre- [équivalent allemand]. (signifiant en français standard, s'il existe)] - renvois à d'autres articles spécification de l'argot: collocation formule de désambiguissation allemande; signification 2 , etc.

Pour signaler le niveau stylistique d'un mot ou son appartenance à un sociolecte, Villatte se sert de symboles déjà utilisés dans la grande Enzyklopädie Sachs-Villatte. Ses marquages sont :

familier ; populaire ; langue du peuple ordinaire (des gewöhnlichen Volkes); 1. des voyous et des voleurs; militaire; marine; commerce; franc-maçonnerie; néologisme ; rare, peu usuel ; vieilli. (Meyer-Riefstahl \& Flandin 1912 : XV, trad. par F.-J. M.)

On ne sera pas surpris que l'inventaire des Parisismen exprime le langage de son époque. L'écart avec le registre correspondant de nos jours touche bien sûr les deux langues concernées. Pour le français, la plupart des lemmes et des collocations sont tombés en désuétude ; et, en grande partie, un francophone sexagénaire ne les comprend plus. On constate le même phénomène pour les équivalents allemands. Cette évolution n'est évidemment pas seulement due au fait "que la langue bouge", mais que ces mouvements de la langue suivent les changements du monde, à savoir de la vie professionnelle, des mœurs, des modes de vie, etc. Ainsi, l'argot des métiers qu'on trouve encore dans les Parisismen est une langue de spécialité disparue avec les petits ateliers d'antan et leurs artisans (Duneton dans Meissner 1999 : 123).

\subsection{Analyse pédagogique des Parisismen}

Confectionner un dictionnaire bilingue signifie qu'on a affaire à l'intersynonymie. Selon Schaeder (1990: 71), une équivalence de signification totale demande une congruence sémantique absolue aux niveaux paradigmatique, syntagmatique et diasystémique ( = diachronique, diatopique, diastratique, diaconnotatif, diatechnique, dianormatif, diafréquent) des mots ou collocations correspondants appartenant à au moins deux langues ou variétés différentes. La définition implique que, hormis les créations monosémiques des langues de spécialité du type bistouri, une intersynonymie totale n'existe que très rarement. Pour la lexicographie bilingue du substandard, le problème est particulièrement délicat. Dans le contraste français-allemand, il concerne de très nombreuses asymétries diasystémiques. Tandis que le français a un registre colloquial commun (hexagonal) avec une nette prépondérance de la variété parisienne, celui de l'allemand est régionalement très fortement varié. Par conséquent, un Munichois traduirait un mot du français non-conventionnel souvent autrement qu'un Hambourgeois. Pourtant, tous deux ont une connaissance passive des dialectes usuels du diaregistre allemand. La problématique est également assez virulente sur le plan de la variation diafréquente. Jusqu'à nos jours, la lexicographie bilingue des parlers colloquiaux ou non-conventionnels n'a pas de réponse à ce problème9.

La problématique est illustrée par trois exemples. Exemple 1: La traduction de la formule vulgaire je pisserai sur ta fosse par mit deinen Knochen werfe ich noch Nüsse herunter (ich werde dich noch lange überleben) est inadéquate et critiquable pour plusieurs raisons : (1) au niveau stylistique, la traduction allemande ne correspond pas à la formule française ; (2) elle est cryptique et à tel point incompréhensible qu'elle demande à être explicitée par l'autre formule «je te survivrai». Une traduction stylistiquement 
adéquate aurait été ich werd' auf dein Grab pissen. (3) De plus, la formule française est parfaitement transparente pour le germanophone à condition qu'il connaisse les mots utilisés (ce qui est probable à l'exception de fosse). (4) Notons qu'il est d'ailleurs discutable de savoir s'il s'agit vraiment d'une formule argotique ou simplement d'un parler de mauvais goût. Une vulgarité verbale n'est pas forcément une spécificité de l'argot ou du parisien. (5) Pour l'étranger, il n'est pas du tout clair que la collocation soit du type figé comme dormir à la belle étoile (principe idiomatique) ou soit une combinaison libre (open choice principle). On constate de pareils phénomènes pour l'exemple 2: se pisser 'fillette qui commence à se sentir' seit kurzer Zeit mannbares Mädchen ( = jeune fille qui vient d'atteindre l'âge nubile). Une fois de plus, l'explication est cryptique, et la désambiguïsation allemande (stylistiquement très recherchée) trahit une tendance à contraster le «bas langage (français) » à un allemand cultivé. Estce l'expression du regard méprisant du bourgeois (allemand) sur le bas peuple (français) et son langage? En tout cas, ne pas traduire une formule vulgaire (et socialement dangereuse) par un équivalent stylistique augmente considérablement le risque du « faux pas » de l'hétéroglotte en communication interculturelle.

En ce qui concerne les argots spéciaux, les auteurs cherchent très loin: PIQUE-CHIEN Portier der polytechnischen Schule, GAUDIN Dieb, Räuber, GENOULELÈTE Rebhuhn. De tels exemples - cités au hasard - montrent que les auteurs n'ont aucun critère pertinent au niveau de la sélection du vocabulaire. La $8^{\mathrm{e}}$ édition a largement dépassé le nombre d'enregistrements des dictionnaires-sources (d'un Delvau, Larchey ou Rigaud) utilisés par Villatte dans les années $1880^{10}$. Mais il ne faut pas oublier qu'entre 1884 et 1912, la France a vu foisonner les dictionnaires d'argot. Nos observations sont par conséquent temporaires. Une étude plus vaste serait nécessaire pour détecter les changements des inventaires des différentes refontes et de leur dépendance de la lexicographie du français non-conventionnel de l'époque.

Somme toute, les Parisismen de 1912 qui reflètent un désir de connaître - si possible tous les mots et collocations recueillis quelque part sous le terme " argot de Paris »-, avaient brisé le cadre des possibilités d'un apprentissage dirigé ${ }^{11}$.

\section{L'impact des Parisismen sur les cours de français en Allemagne}

Il va sans dire que les Parisismen de Villatte ont laissé peu de traces dans les manuels de français en usage outre-Rhin. Les principales raisons en ont été mentionnées: instructions encore et toujours orientées sur des textes écrits, subordination exagérée de l'oral à l'écrit, incompréhension pour les implications psycholinguistiques de l'oral, une linguistique qui n'avait pas encore vraiment découvert la langue parlée, mépris pour la communication de tous les jours, impossibilité technique de capter la langue parlée authentique, last but not least de très nombreuses connotations sexuelles, criminelles et scatologiques attribuées à ce registre et sa présentation dans les dictionnaires spécialisés.

La situation allait changer quand la didactique des langues étrangères, entretemps munie de technologies pour capter la langue parlée authentique et présenter l'oral dans les cours de langue portant un regard plus différencié sur la langue parlée, s'est voulu expressément de plus en plus « communicative ». Pour ne citer, à l'égard du FLE., 
que quelques dates importantes de cette évolution : élaboration du Français fondamental et découverte de l'oral pour la compétence communicative interculturelle, méthode structuro-globale audio-visuelle, approche communicative sous ses différentes facettes, support fourni par les TICES, apprentissage des langues en partenariat (tandem). Les manuels scolaires actuels du français en Allemagne se sont ouverts au français de tous les jours - filtrés certainement par des critères de bon sens pédagogique (adéquation et faisabilité pédagogiques, choix du vocabulaire selon l'utilité) -, cette fois dirigés par un souhait de préparer les élèves aux situations de communication interculturelle.

Nul doute que les Parisismen de Villatte marquent une étape importante dans cette évolution.

\section{Extrait des Parisismen}

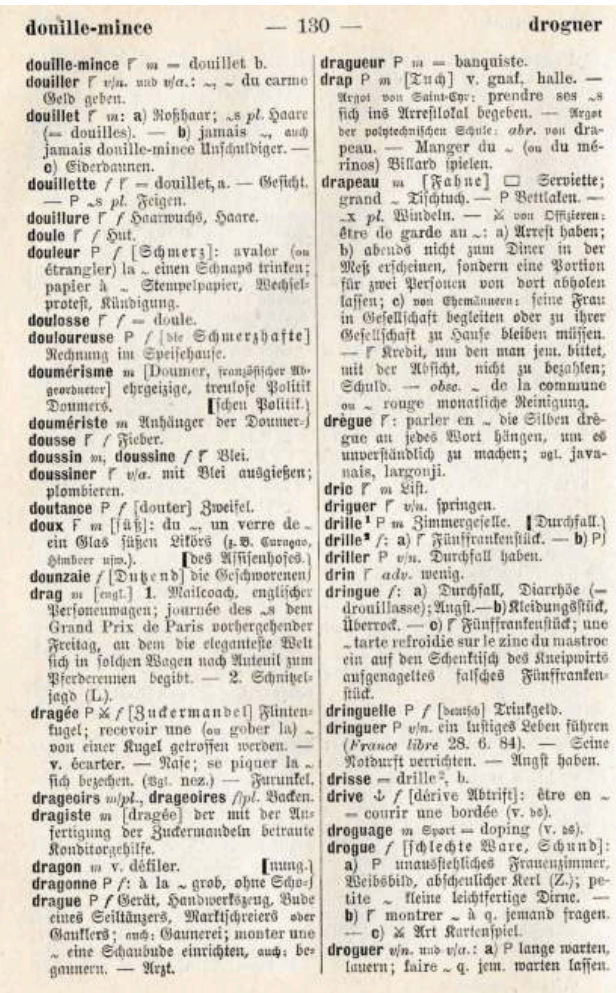

\section{BIBLIOGRAPHIE}

\section{Sources primaires}

VILLATTE, Césaire (1884). Parisismen. Alphabetisch geordnete Sammlung der eigenartigen Ausdrucksweisen des Pariser Argot. Ein Supplement zu allen französisch-deutschen Wörterbüchern. Berlin : Langenscheidt. 
VILLATTE, Césaire (1912). Parisismen. Alphabetisch geordnete Sammlung der eigenartigen Ausdrücke des Pariser Argot, neubearbeitet von Rudolf Meyer-Riefstahl und Marcel Flandin, Berlin-Schöneberg : Langenscheidt.

\section{Sources secondaires}

BAUCHE, Henri (1920). Le langage populaire. Grammaire, syntaxe et dictionnaire du français, tel qu'on le parle dans le peuple de Paris. Avec tous les termes de l'argot usuel. Paris : Payot. BOTт, Gerhard (1982). Deutsche Frankreichkunde 1900-1933. Rheinfelden : Schäuble.

CASCIANI, Clément (1894). « Histoire de l'Argot ». In La Rue, 5-54.

CELLARD, Jacques, REY, Alain (1981). Dictionnaire du français non-conventionnel. Paris : Masson/ Hachette.

CHRIST, Herbert (2005). « Du maître de langue au Neuphilologe. La formation des enseignants de français en Allemagne au cours du XIX ${ }^{\mathrm{e}}$ siècle ». Documents pour l'histoire du français langue étrangère et seconde 33/34. In Brigitte Lépinette, Maria Elena Jiménez \& Julia Pinilla, J. (éds). L'enseignement du français en Europe autour du XIX ${ }^{\mathrm{e}}$ siècle. Histoire professionnelle et sociale, 47-62.

CHRIST, Herbert \& RANG, Joachim (Hrsg.) (1985). Fremdsprachenunterricht unter staatlicher Verwaltung 1700 bis 1945, Dokumentation amtlicher Richtlinien und Verordnungen. Tübingen : Narr, 7 vols.

ESNAULT, Gaston (1965). Dictionnaire historique des argots français. Paris : Larousse.

FRÄNKEL Ludwig. « Villatte, Césaire ». Allgemeine Deutsche Biographie 39 (1895), 705-707. En

ligne : <http://www.deutsche-biographie.de/pnd117418196.html?anchor=adb> (14 avril 2016)

GREIVE, Artur (1984). « Remarques sur l'histoire du français parlé ». Cahiers de I’Institut de Linguistique de Louvain 10, 65-76.

LA RUE, Jean (1986) [1884]. Dictionnaire d'Argot et des principales locutions populaires. Précédé d'une Histoire de l'Argot par Clément Casciani. Paris : Flammarion.

LARCHEY, Lorédan (1985) [1872]. Dictionnaire historique, étymologique et anecdotique de l'Argot parisien. Sixième édition des Excentricités du langage, mise à la hauteur des révolutions du jour. Les Éditions de Paris.

LaWUF = MEISSNER, Franz-Joseph, LEGRAND, Jean-Paul, LENOIR, Roger, MEISSNER, Claude (1992).

Langenscheidts Wörterbuch der Umgangssprache : Französisch. Munich : Langenscheidt ( $3^{\mathrm{e}}$ éd. 1994).

MEISSNER, Franz-Joseph (1987). « Der französische Sprechwortschatz nach lexikodidaktischen Gebrauchsklassen ». Die Neueren Sprachen 86, 554-568.

MEISSNER, Franz-Joseph (2008). « Diastratische und diaphasische Varietäten des Französischen ». In Ingo Kolboom, Thomas Kotschi \& Edward Reichel (Hrsg.) : Handbuch Französisch. Lehre Studium - Praxis. Berlin : Schmid, 87-92.

MEISSNER, Franz-Joseph (1999). « Entre l'argot et la tchatche - Où en est-on en français parlé ? Entretien avec Claude Duneton ». Französisch heute 30, 120-131. 
SCHAEDER, B. (1990). « Das Problem der Äquivalenz aus Sicht der Internationalismenforschung ». In Peter Braun, Burkhard Schaeder \& Johannes Volmert (Hrsg.). Internationalismen. Studien zur interlingualen Lexikologie und Lexikographie. Tübingen : Niemeyer, 63-73.

SCHIFRES, A. (1990). Les Parisiens. Paris : Lattès.

SCHRÖDER, Konrad (1989). « Über Volkshaß und über den Gebrauch einer fremden Sprache. Zur historischen Dimension des Schulsprachenstreites Englisch - Französisch, unter besonderer Berücksichtigung der nach-Napoleonischen Zeit ». In Eberhard Kleinschmidt (Hrsg.). Fremdsprachenunterricht zwischen Sprachenpolitik und Praxis. Festschrift für Herbert Christ zum 60. Geburtstag. Tübingen : Narr, 58-70.

söLL, Ludwig \& HAUSMANN, Franz Josef (1974). Gesprochenes und geschriebenes Französisch. Berlin : Schmidt (1985, 3. verb. Aufl.).

VIËTOR, W. (1882). Der Sprachunterricht muß umkehren von Quousque Tandem. Ein Beitrag zur Überbürdungsfrage. Heilbronn : Henninger (Wiederabdruck der 3. Aufl. 1905 : mit einer Einleitung von Konrad Schröder, Munich : Hueber).

VOSSLER, Karl (1922). « Vom Bildungswert der romanischen Sprachen. Vortrag gehalten auf dem 18. Neuphilologentag in Nürnberg am 7. Juni 1922 ». Die Neueren Sprachen 30, 226-234.

\section{NOTES}

1. Cet intérêt se manifeste d'abord dans des cours chargés de thématiques romanes (Diez, Schlegel, von Maffei et d'autres) dans les années 1820, ensuite dans la fondation de chaires de philologie romane (1830 Bonn, 1833 Halle, 1844 Tübingen, 1836 Marburg, 1862 Leipzig, 1870 Berlin) et, last but not least, dans de nouvelles revues (1846 Archiv für das Studium der neueren Sprachen, 1859 Jahrbuch für romanische und englische Literatur, 1877 Zeitschrift für Romanische Philologie, 1883 Romanische Forschungen, etc.).

2. Mais quand, par contre, vous parlez françois/Faut pas que cela ressemble à du chinois/Träng (train), Detalch (détail) et Reglemang (règlement)/En voilà de drôles de sons. (Trad. par F.-J. M.)

3. Que l'on compare seulement, de manière diachronique, les articles Peuples (all. Pöbel « populace ») et Volk dans les « lexiques » de grande diffusion entre 1880 et 1960.

4. Les manuels expliquent, en général, la prononciation des sons et des mots de manière plus ou moins scrupuleuse mais parlent peu de l'euphonie au niveau de la phrase.

5. Il s'agit d'une note en bas de page.

6. Villatte se réfère ici à ceux de Dervau, Larchey, Rigaud.

7. Französische Lieder aus der Florentiner Handschrift Strozzi-Magliabecchiana CL.VII.1040: Versuch einer kritischen Ausgabe; publication: Tübingen 1907.

8. Qu'on compare l'article DRAP: « drap P $m$ [Tuch] v. gnaf, halle. - Argot von Saint-Cyr: prendre ses s sich ins Arrestlokal begeben ». "Lemme - niveau stylistique - genre - [équivalent allemand du mot en français standard] - ».

9. Les auteurs du LaWUF (1992) ont régulièrement consulté des dialectophones de plusieurs variétés de l'allemand pour choisir l'équivalent d'un enregistrement non-conventionnel français. 10. Ces mots et tant d'autres des Parisismen sont absents de la liste de Bauche (1920:181) qui prétend contenir «tous les mots et locutions propres au langage populaire parisien, employés couramment et habituellement ».

11. Rappelons qu'entre 1884 et 1912 , la lexicographie ne disposait pas encore de dictionnaires de fréquence. À titre de comparaison : sur la base de plusieurs dictionnaires actuels du français nonconventionnel et à partir des informations introspectives fournies par plus de 30 francophones 
de tous âges et régions confondus, le LaWUF, qui totalise 8000 enregistrements français nonconventionnels, sélectionne ses lemmes et les repartit en quatre «catégories d'usage ». Quant à la fiabilité statistique de la méthode introspective utilisée, voir Meissner (1987).

\section{RÉSUMÉS}

L'article décrit le rôle joué par le dictionnaire des Parisismen de Césaire Villatte (1884 et sq.) dans l'apprentissage-enseignement $\mathrm{du}$ français dans les pays de langue germanique. Le terme historique de parisianismes prête facilement à confusion étant donné que différents registres et styles peu délimités étaient concernés (parlé, courant, familier, populaire, argot, vulgaire, etc.). Au temps de Villatte, l'enseignement du français, en particulier dans les écoles allemandes, visait surtout la langue écrite et les textes provenaient des plus illustres auteurs de la pensée française. Quand des auteurs naturalistes de renom ont intégré le langage du peuple dans leurs romans, cela a changé le statut du français "parlé », ce que de nombreux dictionnaires d'argot de l'époque documentent. En Allemagne, cette évolution a coïncidé avec des réformes curriculaires soulignant l'importance de la compétence communicative, situation qui explique l'ouvrage de Villatte.

The paper describes the role of Césaire Villatte's Parisismen dictionary (1884 et seq.) for the teaching and learning of French in German speaking countries. The historic term parisisms easily leads to confusion, as it covered various linguistic registers and styles of informal speech whose extents were quite fuzzy (spoken, colloquial, argot, familiar, popular, vulgar or rude, etc.). In the days of Villatte, the teaching of French, especially in German schools, focused on the written language and its texts were mainly provided by the illustrious authors of French thought. But when the renowned literary naturalism integrated the lower classes' speech in its works, this changed the status of spoken French, which is documented by numerous 'argot' dictionaries. In Germany, this change coincided with syllabus reforms stressing the importance of communicative competence. The situation makes clear why Villatte composed a dictionary focusing the Parisismen.

\section{INDEX}

Mots-clés : enseignement-apprentissage du français, dictionnaires allemands du français parlé $\mathrm{du}$ XIXe siècle, parisianismes, argot, français parlé en Allemagne

Keywords : Teaching and learning French, 19th century German dictionaries of spoken French, parisisms, argot, spoken French in Germany

\section{AUTEUR}

\section{FRANZ-JOSEPH MEISSNER}

Université Justus Liebig de Giessen

franz-joseph.meissner@sprachen.uni-giessen.de 